\title{
The Step-Type Contrast Structure for High Dimensional Tikhonov System with Neumann Boundary Conditions
}

\author{
Aifeng Wang ${ }^{1}$ and Mingkang $\mathrm{Ni}^{2}$ \\ ${ }^{1}$ School of Mathematical Science, Huaiyin Normal University, Huaian 223001, China \\ ${ }^{2}$ Department of Mathematics, East China Normal University, Shanghai 200062, China \\ Correspondence should be addressed to Aifeng Wang; waf2003@126.com
}

Received 24 November 2015; Revised 11 January 2016; Accepted 27 January 2016

Academic Editor: Douglas R. Anderson

Copyright (c) 2016 A. Wang and M. Ni. This is an open access article distributed under the Creative Commons Attribution License, which permits unrestricted use, distribution, and reproduction in any medium, provided the original work is properly cited.

\begin{abstract}
We investigate the step-type contrast structure for high dimensional Tikhonov system with Neumann boundary conditions. We not only propose a key condition with the existence of the number of mutually independent first integrals under which there exists a step-type contrast structure, but also determine where an internal transition time is. Using the method of boundary function, we construct the formal asymptotic solution and give the analytical expression for the higher order terms. At the same time, the uniformly valid asymptotic expansion and the existence of such an available step-type contrast structure are obtained by sewing connection method.
\end{abstract}

\section{Introduction}

The fundamental characteristic of contrast structure is that there is $t^{*}$ (or multiple $t^{*}$ ) within the domain of interest, which is called an internal transition point. The position of $t^{*}$ which is unknown in advance needs to be determined thereafter. In the neighborhood of $t^{*}$, the genuine solution will have an abrupt structure change and in the different side of $t^{*}$, it will approach different reduced solutions when the small parameter $\mu \rightarrow 0$. The contrast structure in singularly perturbed problems is classified as a step-type contrast structure [1-6] or a spike-type contrast structure [79]. In the West, the study on this issue is mainly by the method of dynamic systems or geometric method [10-12]. In recent years, the study on contrast structures is still a hot but difficult research topic in the theory of singularly perturbed problem, especially for high dimensional singularly perturbed system [13]. In fact, the existence of a step-type contrast structure is closely related to the existence of a heteroclinic orbit of its auxiliary system in its corresponding phase space. However, how to find and construct such an orbit in a high dimensional dynamic is itself difficult in general in the theory of qualitative analysis. This is why it is nontrivial in extending to high dimensional case from the contrast structures in plane. Using the method of boundary function and sewing connection, $\mathrm{Ni}$ and Wang [14] investigate the step-type contrast structure for the following singular perturbed system:

$$
\begin{aligned}
\mu \frac{d y}{d t} & =f(y, t) ; \\
A y(0, \mu) & =A y_{0}, \\
B y(1, \mu) & =B y_{1},
\end{aligned}
$$

where $y, f$ are $n$-dimensional vectors. In this paper, we extend the existence of step-type contrast structure to the following:

$$
\begin{aligned}
\mu \frac{d z}{d t} & =f(z, y, t), \\
\frac{d y}{d t} & =g(z, y, t),
\end{aligned}
$$

$$
0 \leq t \leq 1
$$

$$
\begin{aligned}
A z^{\prime}(0, \mu)+B z^{\prime}(1, \mu) & =z^{0}, \\
C y(0, \mu)+D y(1, \mu) & =y^{0},
\end{aligned}
$$

where $\mu>0$ is a small parameter, $z, f$ are $M$-dimensional vectors, $y, g$ are $m$-dimensional vectors, $A=\left(\begin{array}{cc}E_{k} & 0 \\ 0 & 0\end{array}\right)$, 
$B=\left(\begin{array}{cc}0 & 0 \\ 0 & E_{M-k}\end{array}\right)$ are $M \times M$ matrixes, $E_{k}$ is an $k \times k$ unit matrix, and $E_{M-k}$ is an $(M-k) \times(M-k)$ unit matrix. $C=\left(\begin{array}{cc}E_{h} & 0 \\ 0 & 0\end{array}\right)$, $D=\left(\begin{array}{cc}0 & 0 \\ 0 & E_{m-h}\end{array}\right)$ are $m \times m$ matrixes, $E_{h}$ is an $h \times h$ unit matrix, and $E_{m-h}$ is an $(m-h) \times(m-h)$ unit matrix.

\section{Assumptions}

The following assumptions are fundamental in theory for the problem in question.

$\left(H_{1}\right)$ Suppose that $f(z, y, t)$ and $g(z, y, t)$ are sufficiently smooth on the domain $D=\left\{(z, y, t):|z| \leq a_{1},|y| \leq a_{2}, 0 \leq\right.$ $t \leq 1\}$, where $a_{1}, a_{2}$ are real numbers.

The reduced system of (2) is given by

$$
\begin{aligned}
f(\bar{z}, \bar{y}, t) & =0, \\
\frac{d \bar{y}}{d t} & =g(\bar{z}, \bar{y}, t) .
\end{aligned}
$$

$\left(\mathrm{H}_{2}\right)$ Suppose that the reduced subsystem of (2) given by $f(\bar{z}, \bar{y}, t)=0$ has two isolated solutions on $\bar{D}: \bar{z}=$ $\alpha(\bar{y}, t)$ and $\bar{z}=\beta(\bar{y}, t)$, where $\alpha=\left(\alpha_{1}, \alpha_{2}, \ldots, \alpha_{M}\right)^{T}, \beta=$ $\left(\beta_{1}, \beta_{2}, \ldots, \beta_{M}\right)^{T}$.

$\left(H_{3}\right)$ For $0 \leq t \leq 1$, suppose that $f_{z}\left(\alpha\left(\bar{y}^{(-)}(t), t\right), \bar{y}^{(-)}(t), t\right)$ and $f_{z}\left(\beta\left(\bar{y}^{(+)}(t), t\right), \bar{y}^{(+)}(t), t\right)$ have characteristic roots $\bar{\lambda}_{i}^{1}(t)$ and $\bar{\lambda}_{i}^{2}(t),(i=1,2, \ldots, M)$, respectively, which satisfy

$$
\begin{aligned}
& \operatorname{Re} \bar{\lambda}_{i}^{j}(t)<0, \quad i=1,2, \ldots, k<M \\
& \operatorname{Re} \bar{\lambda}_{i}^{j}(t)>0, \quad i=k+1, k+2, \ldots, M,
\end{aligned}
$$

where $j=1,2$.

$\left(H_{3}\right)$ shows that, for fixed $\bar{t} \in[0,1]$, the auxiliary system given by

$$
\frac{d \widetilde{z}}{d \tau}=f(\widetilde{z}, \bar{y}(\bar{t}), \bar{t})
$$

has two equilibriums $\widetilde{z}=\alpha\left(\bar{y}^{(-)}(\bar{t}), \bar{t}\right)$ and $\widetilde{z}=\beta\left(\bar{y}^{(+)}(\bar{t}), \bar{t}\right)$ which are all hyperbolic saddle points.

\section{Construction of the Asymptotic Expansion}

Let $t^{*} \in(0,1)$ be the transition point and it is given by

$$
t^{*}=t_{0}+\mu t_{1}+\mu^{2} t_{2}+\cdots+\mu^{k} t_{k}+\cdots,
$$

with $t_{k}(k=0,1, \ldots)$ which will be given in the following.

Suppose that the asymptotic solution is composed of two parts.

The left problem $\left(0 \leq t \leq t^{*}\right)$ :

$$
\begin{aligned}
\mu \frac{d z^{(-)}}{d t} & =F\left(z^{(-)}, y^{(-)}, t\right), \\
\frac{d y^{(-)}}{d t} & =f\left(z^{(-)}, y^{(-)}, t\right) ;
\end{aligned}
$$

$$
\begin{gathered}
A z^{(-)^{\prime}}(0, \mu)=A z^{0}, \\
B z^{(-)}\left(t^{*}, \mu\right)=B z^{*}, \\
C y^{(-)}(0, \mu)=C y^{0}, \\
D y^{(-)}\left(t^{*}, \mu\right)=D y^{*} .
\end{gathered}
$$

The right problem $\left(t^{*} \leq t \leq 1\right)$ :

$$
\begin{aligned}
\mu \frac{d z^{(+)}}{d t} & =F\left(z^{(+)}, y^{(+)}, t\right), \\
\frac{d y^{(+)}}{d t} & =f\left(z^{(+)}, y^{(+)}, t\right) ; \\
A z^{(+)}\left(t^{*}, \mu\right) & =A z^{*}, \\
B z^{(+)^{\prime}}(1, \mu) & =B z^{1}, \\
C y^{(+)}\left(t^{*}, \mu\right) & =C y^{*}, \\
D y^{(+)}(1, \mu) & =D y^{1},
\end{aligned}
$$

where $x^{*}=\left(z^{*}, y^{*}\right)^{T}$ are parameters which will be determined in the following but related to $t^{*}$. For convenience, let $x^{*}=x_{0}^{*}+\mu x_{1}^{*}+\cdots$.

To obtain the step-type solution, we need

$$
x^{(-)}\left(t^{*}, \mu\right)=x^{(+)}\left(t^{*}, \mu\right) .
$$

Let $x=(y, z)^{T}$. Suppose the formal asymptotic solutions for the left and the right problem are

$$
\begin{aligned}
& x^{(-)}(t, \mu)=\sum_{j=0}^{\infty} \mu^{j}\left(\bar{x}_{j}^{(-)}(t)+L_{j} x\left(\tau_{0}\right)+Q_{j}^{(-)} x(\tau)\right), \\
& x^{(+)}(t, \mu)=\sum_{j=0}^{\infty} \mu^{j}\left(\bar{x}_{j}^{(+)}(t)+Q_{j}^{(+)} x(\tau)+R_{j} x\left(\tau_{1}\right)\right),
\end{aligned}
$$

respectively, where $\tau_{0}=t / \mu>0, \tau=\left(t-t^{*}\right) / \mu$, and $\tau_{1}=(t-1) / \mu<0 . \bar{x}_{i}^{(\mp)}(t)$ are coefficients of regular terms; $L_{j} x\left(\tau_{0}\right)$ are coefficients of the boundary layer terms at $t=0$; $R_{j} x\left(\tau_{1}\right)$ are coefficients of the boundary layer terms at $t=$ $1 ; Q_{j}^{(\mp)} x(\tau)$ are the left and the right coefficients of internal transition terms at $t=t^{*}$. Furthermore, $\lim _{\tau_{0} \rightarrow+\infty} L_{j} x\left(\tau_{0}\right)=$ $0, \lim _{\tau \rightarrow \mp \infty} Q_{j}^{(\mp)} x(\tau)=0$, and $\lim _{\tau_{1} \rightarrow-\infty} R_{j} x\left(\tau_{1}\right)=0(j=$ $0,1, \ldots)$.

3.1. Construction of the Zero-Order Terms. Substituting (15), (16) into (8)-(10) and (11)-(13), respectively, by the boundary function method [15], we have

$$
f\left(\bar{z}_{0}^{(\mp)}(t), \bar{y}_{0}^{(\mp)}(t), t\right)=0 .
$$

By $\left(H_{2}\right)$ and the assumed solution type, we know

$$
\begin{aligned}
& z_{0}^{(-)}(t)=\alpha\left(\bar{y}_{0}^{(-)}(t), t\right), \\
& z_{0}^{(+)}(t)=\beta\left(\bar{y}_{0}^{(+)}(t), t\right),
\end{aligned}
$$


while $\bar{y}_{0}^{(\mp)}(t)$ satisfy the following problems:

$$
\begin{aligned}
\frac{d \bar{y}_{0}^{(-)}(t)}{d t} & =g\left(\alpha\left(\bar{y}_{0}^{(-)}(t), t\right), \bar{y}_{0}^{(-)}(t), t\right) ; \\
C \bar{y}_{0}^{(-)}(0) & =C y^{0}, \\
D \bar{y}_{0}^{(-)}\left(t_{0}\right) & =D y_{0}^{*}, \\
\frac{d \bar{y}_{0}^{(+)}(t)}{d t} & =g\left(\beta\left(\bar{y}_{0}^{(+)}(t), t\right), \bar{y}_{0}^{(+)}(t), t\right) ; \\
C \bar{y}_{0}^{(+)}\left(t_{0}\right) & =C \bar{y}_{0}^{*}, \\
D \bar{y}_{0}^{(+)}(1) & =D \bar{y}^{1},
\end{aligned}
$$

respectively.

$\left(H_{4}\right)$ Suppose the solution of (19), (20) and the solution of (21), (22) are transversal at $y_{0}^{*}$, where $y_{0}^{*}=y_{0}^{*}\left(t_{0}\right)$.

Next, we give the equations and their conditions for determining the zero-order coefficient of $Q_{0}^{(\mp)} x(\tau)$ as follows:

$$
\begin{aligned}
& \frac{d Q_{0}^{(-)} z}{d \tau} \\
& \quad=f\left(\alpha\left(t_{0}\right)+Q_{0}^{(-)} z(\tau), \bar{y}_{0}^{(-)}\left(t_{0}\right)+Q_{0}^{(-)} y(\tau), t_{0}\right) \\
& \frac{d Q_{0}^{(-)} y}{d \tau}=0 \\
& B\left(\bar{z}_{0}^{(-)}\left(t_{0}\right)+Q_{0}^{(-)} z(0)\right)=B z_{0}^{*}, \\
& Q_{0}^{(-)} x(-\infty)=0 \\
& \frac{d Q_{0}^{(+)} z}{d \tau} \\
& \quad=f\left(\beta\left(t_{0}\right)+Q_{0}^{(+)} z(\tau), \bar{y}_{0}^{(+)}\left(t_{0}\right)+Q_{0}^{(+)} y(\tau), t_{0}\right) \\
& \frac{d Q_{0}^{(+)} y}{d \tau}=0 ; \\
& A\left(\bar{z}_{0}^{(+)}\left(t_{0}\right)+Q_{0}^{(+)} z(0)\right)=A z_{0}^{*}, \\
& Q_{0}^{(+)} x(+\infty)=0 .
\end{aligned}
$$

Let $\alpha\left(t_{0}\right)+Q_{0}^{(-)} z(\tau)=\widetilde{z}^{(-)}(\tau), \beta\left(t_{0}\right)+Q_{0}^{(+)} z(\tau)=\widetilde{z}^{(+)}(\tau)$. Then we have

$$
\begin{aligned}
\frac{d \widetilde{z}^{(-)}}{d \tau} & =f\left(\widetilde{z}^{(-)}, \bar{y}_{0}^{(-)}\left(t_{0}\right), t_{0}\right) ; \\
B \widetilde{z}^{(-)}(0) & =B z_{0}^{*}, \\
\tilde{z}^{(-)}(-\infty) & =\alpha\left(t_{0}\right),
\end{aligned}
$$

$$
\begin{aligned}
\frac{d \widetilde{z}^{(+)}}{d \tau} & =f\left(\widetilde{z}^{(+)}, \bar{y}_{0}^{(+)}\left(t_{0}\right), t_{0}\right) ; \\
A \widetilde{z}^{(+)}(0) & =A z_{0}^{*}, \\
\widetilde{z}^{(+)}(+\infty) & =\beta\left(t_{0}\right) .
\end{aligned}
$$

Obviously, $M_{-}\left(\alpha\left(t_{0}\right), \bar{y}^{(-)}\left(t_{0}\right)\right)$ is a hyperbolic saddle point of (29) and $M_{+}\left(\beta\left(t_{0}\right), \bar{y}^{(+)}\left(t_{0}\right)\right)$ is a hyperbolic saddle point of (30).

The existence of the solutions for (23)-(28) will be given in the following. If they are used in the following calculation process, we think that they are known. Obviously, they are associated with $t_{0}$. Moreover, by the boundary conditions $L_{0}^{\prime} z(0)=0, R_{0}^{\prime} z(0)=0$ and $L_{0} x(+\infty)=0, R_{0} x(-\infty)=0$, we have $L_{0} z\left(\tau_{0}\right) \equiv 0, R_{0} z\left(\tau_{1}\right) \equiv 0$.

3.2. Construction of the Higher Order Terms. For $\bar{x}_{j}^{(\mp)}(t)$, we have the equations and their boundary conditions as follows:

$$
\begin{aligned}
& \frac{d \bar{z}_{j-1}^{(-)}(t)}{d t} \\
& =\bar{f}_{z}^{(-)}(t) \bar{z}_{j}^{(-)}(t)+\bar{f}_{y}^{(-)}(t) \bar{y}_{j}^{(-)}(t)+f_{j}^{(-)}(t), \\
& \frac{d \bar{y}_{j}^{(-)}(t)}{d t} \\
& =\bar{g}_{z}^{(-)}(t) \bar{z}_{j}^{(-)}(t)+\bar{g}_{y}^{(-)}(t) \bar{y}_{j}^{(-)}(t)+g_{j}^{(-)}(t) ; \\
& \bar{C}\left(\bar{y}_{j}^{(-)}(0)+L_{j} y(0)\right)=0, \\
& D\left(\bar{y}_{0}^{(-)}\left(t_{0}\right) t_{j}+\gamma^{(-)}+Q_{j}^{(-)} y(0)\right)=D y_{j}^{*}, \\
& \frac{d \bar{z}_{j-1}^{(+)}(t)}{d t} \\
& =\bar{f}_{z}^{(+)}(t) \bar{z}_{j}^{(+)}(t)+\bar{f}_{y}^{(+)}(t) \bar{y}_{j}^{(+)}(t)+f_{j}^{(+)}(t), \\
& \frac{d \bar{y}_{j}^{(+)}(t)}{d t} \\
& =\bar{g}_{z}^{(+)}(t) \bar{z}_{j}^{(+)}(t)+\bar{g}_{y}^{(+)}(t) \bar{y}_{j}^{(+)}(t)+g_{j}^{(+)}(t) ; \\
& C\left(\bar{y}_{0}^{(+)}\left(t_{0}\right) t_{j}+\gamma^{(+)}+Q_{j}^{(+)} y(0)\right)=C y_{j}^{*}, \\
& D\left(\bar{y}_{j}^{(+)}(1)+R_{j} y(0)\right)=0,
\end{aligned}
$$

where $\bar{f}_{z}^{(-)}(t), \bar{f}_{z}^{(+)}(t)$ take value at $\left(\alpha(t), \bar{y}_{0}^{(-)}(t), t\right)$ and $\left(\beta(t), \bar{y}_{0}^{(+)}(t), t\right)$, respectively. $\bar{f}_{y}^{(\mp)}(t), \bar{g}_{z}^{(\mp)}(t)$, and $\bar{g}_{y}^{(\mp)}(t)$ have the same significance. $f_{j}^{(\mp)}(t), g_{j}^{(\mp)}(t)$ are known functions and $\gamma_{j}^{(\mp)}$ are determined. 
$L_{j} x\left(\tau_{0}\right)$ satisfy the following problems:

$$
\begin{aligned}
& \frac{d L_{j} z}{d \tau_{0}}=\tilde{f}_{z}^{(l)}\left(\tau_{0}\right) L_{j} z+\tilde{f}_{y}^{(l)}\left(\tau_{0}\right) L_{j} y+H_{j}^{(l)}\left(\tau_{0}\right), \\
& \frac{d L_{j} y}{d \tau_{0}}=L_{j-1} g\left(\tau_{0}\right) ; \\
& A\left(\bar{z}_{j-1}^{(-)^{\prime}}(0)+L_{j} z(0)\right)=0, \\
& L_{j} x(+\infty)=0,
\end{aligned}
$$

where $\widetilde{f}_{x}^{(l)}\left(\tau_{0}\right)=f_{x}\left(\alpha(0)+L_{0} z, \bar{y}_{0}^{(-)}(0), 0\right) . H_{j}^{(l)}\left(\tau_{0}\right), L_{j-1} g\left(\tau_{0}\right)$ are determined functions.

By (38) and $L_{j} y(+\infty)=0$, we know that $L_{j} y\left(\tau_{0}\right)=$ $\int_{+\infty}^{\tau_{0}} L_{j-1} g(s) d s$. Then, $L_{j} y(0)=\int_{+\infty}^{0} L_{j-1} g(s) d s$. So we have

$$
C \bar{y}_{j}^{(-)}(0)=-C L_{j} y(0)=\int_{0}^{+\infty} C L_{j-1} g(s) d s .
$$

The equations to determine $R_{j} x\left(\tau_{1}\right)$ are given by

$$
\begin{aligned}
& \frac{d R_{j} z}{d \tau_{1}}=\widetilde{f}_{z}^{(r)}\left(\tau_{1}\right) R_{j} z+\widetilde{f}_{y}^{(r)}\left(\tau_{1}\right) R_{j} y+H_{j}^{(r)}\left(\tau_{1}\right), \\
& \frac{d R_{j} y}{d \tau_{1}}=R_{j-1} g\left(\tau_{1}\right) ; \\
& B\left(\bar{z}_{j-1}^{(+)^{\prime}}(1)+R_{j} z(0)\right)=0, \\
& R_{j} x(-\infty)=0,
\end{aligned}
$$

where $\tilde{f}_{x}^{(r)}\left(\tau_{1}\right)=f_{x}\left(\beta(1)+R_{0} z, \bar{y}_{0}^{(+)}(1), 1\right)$, while $H_{j}^{(r)}\left(\tau_{1}\right)$, $R_{j-1} g\left(\tau_{1}\right)$ are known functions.

By (42) and $R_{j} y(-\infty)=0$, we know that $R_{j} y\left(\tau_{1}\right)=$ $\int_{-\infty}^{\tau_{1}} R_{j-1} g(s) d s$. Then, $R_{j} y(0)=\int_{+\infty}^{0} R_{j-1} g(s) d s$. So we have

$$
D \bar{y}_{j}^{(+)}(1)=-D R_{j} y(0)=\int_{0}^{-\infty} D R_{j-1} g(s) d s .
$$

And then $\bar{x}_{j}^{( \pm)}(t)$ can be obtained.

$\left(H_{5}\right)$ Suppose that the solution of (31), (32) and the solution of (34), (35) are transversal at $y_{j}^{*}(j=1,2, \ldots)$.

The equations to determine $Q_{j}^{(-)} x(\tau)$ are given by

$$
\begin{aligned}
& \frac{d Q_{j}^{(-)} z}{d \tau}= f_{z}^{(-)}(\tau) Q_{j}^{(-)} z+f_{y}^{(-)}(\tau) Q_{j}^{(-)} y \\
&+G_{j}^{(-)}(\tau), \\
& \frac{d Q_{j}^{(-)} y}{d \tau}= Q_{j-1}^{(-)} g(\tau) ; \\
& B Q_{j}^{(-)} z(0)=B z_{j}^{*}-B \bar{z}_{0}^{(-)^{\prime}}\left(t_{0}\right) t_{j}+B \rho_{j}^{(-)}, \\
& Q_{j}^{(-)} x(-\infty)=0 .
\end{aligned}
$$

Here

$$
\begin{aligned}
& G_{j}^{(-)}(\tau)=\left(\Delta f_{z}^{(-)}(\tau) \alpha^{\prime}\left(t_{0}\right)+\Delta f_{y}^{(-)}(\tau)\left(\bar{y}_{0}^{(-)}\left(t_{0}\right)\right)^{\prime}\right. \\
& \left.\quad+\Delta f_{t}^{(-)}(\tau)\right) t_{j}+\bar{G}_{j}^{(-)}(\tau), \\
& f_{x}^{(-)}(\tau)=f_{x}\left(\alpha\left(t_{0}\right)+Q_{0}^{(-)} z(\tau), \bar{y}_{0}^{(-)}\left(t_{0}\right), t_{0}\right) .
\end{aligned}
$$

While

$$
\begin{aligned}
\Delta f_{x}^{(-)}(\tau)= & f_{x}\left(\alpha\left(t_{0}\right)+Q_{0} z, \bar{y}_{0}\left(t_{0}\right), t_{0}\right) \\
& -f_{x}\left(\alpha\left(t_{0}\right), \bar{y}_{0}\left(t_{0}\right), t_{0}\right),
\end{aligned}
$$

$\Delta f_{t}^{(-)}(\tau)$ has the same significance. $\bar{G}_{j}^{(-)}(\tau), Q_{j-1}^{(-)} g(\tau)$, and $\rho_{j}^{(-)}$ are determined, excluding $t_{j}$.

By $Q_{j}^{(-)} y(-\infty)=0$ and (46) we have $Q_{j}^{(-)} y(\tau)=$ $\int_{-\infty}^{\tau} Q_{j-1}^{(-)} g(s) d s$. Substituting them into (45) we obtain $Q_{j}^{(-)} z(\tau)$ under the initial condition (47). Moreover, $Q_{j}^{(-)} z(\tau)$ are related to $t_{j}$.

As for $Q_{j}^{(+)} x(\tau)$, it satisfies the following boundary value problem:

$$
\begin{aligned}
& \frac{d Q_{j}^{(+)} z}{d \tau}= f_{z}^{(+)}(\tau) Q_{j}^{(+)} z+f_{y}^{(+)}(\tau) Q_{j}^{(+)} y \\
&+G_{j}^{(+)}(\tau), \\
& \frac{d Q_{j}^{(+)} y}{d \tau}= Q_{j-1}^{(+)} g(\tau) ; \\
& A Q_{j}^{(+)} z(0)=A z_{j}^{*}-A \bar{z}_{0}^{(+)^{\prime}}\left(t_{0}\right) t_{j}+A \rho_{j}^{(+)}, \\
& Q_{j}^{(+)} x(+\infty)=0 .
\end{aligned}
$$

Here

$$
\begin{aligned}
& G_{j}^{(+)}(\tau)=\left(\Delta f_{z}^{(+)}(\tau) \beta^{\prime}\left(t_{0}\right)+\Delta f_{y}^{(+)}(\tau)\left(\bar{y}_{0}^{(+)}\left(t_{0}\right)\right)^{\prime}\right. \\
& \left.\quad+\Delta f_{t}^{(+)}(\tau)\right) t_{j}+\bar{G}_{j}^{(+)}(\tau), \\
& f_{x}^{(+)}(\tau)=f_{x}\left(\beta\left(t_{0}\right)+Q_{0}^{(+)} z(\tau), \bar{y}_{0}^{(+)}\left(t_{0}\right), t_{0}\right) .
\end{aligned}
$$

While

$$
\begin{aligned}
\Delta f_{x}^{(+)}(\tau)= & f_{x}\left(\beta\left(t_{0}\right)+Q_{0} z, \bar{y}_{0}\left(t_{0}\right), t_{0}\right) \\
& -f_{x}\left(\beta\left(t_{0}\right), \bar{y}_{0}\left(t_{0}\right), t_{0}\right),
\end{aligned}
$$

$\Delta f_{t}^{(+)}(\tau)$ has the same significance. $\bar{G}_{j}^{(+)}(\tau), Q_{j-1}^{(+)} g(\tau)$, and $\rho_{j}^{(+)}$are determined, excluding $t_{j}$.

By $Q_{j}^{(+)} y(+\infty)=0$ and (45) we have $Q_{j}^{(+)} y(\tau)=$ $\int_{+\infty}^{\tau} Q_{j-1}^{(+)} g(s) d s$. Substituting it into (28), we get a first-order linear equation, so $y_{1}^{(+)}(t)$ exists under the initial condition (47). And then $\bar{z}_{j}^{(+)}(t)$ exists. Substituting $Q_{j}^{(+)} y(\tau)$ into 
(46) we obtain $Q_{j}^{(+)} z(\tau)$ under the initial condition (46). Moreover, $Q_{j}^{(-)} z(\tau)$ are related to $t_{j}$.

In the following, we will give the analytical expression for $Q_{j}^{(\mp)} z(\tau)$. Because $Q_{j}^{(\mp)} y(\tau)$ are solved, we rewrite (45), (50) as follows:

$$
\frac{d Q_{j}^{(\mp)} z}{d \tau}=f_{z}^{(\mp)}(\tau) Q_{j}^{(\mp)} z+\widetilde{G}_{j}^{(\mp)}(\tau),
$$

where

$$
\begin{aligned}
& \widetilde{G}_{j}^{(\mp)}(\tau)=f_{y}^{(\mp)}(\tau) Q_{j}^{(\mp)} y+\left(\Delta f_{z}^{(\mp)}(\tau) \beta^{\prime}\left(t_{0}\right)\right. \\
& \left.\quad+\Delta f_{y}^{(\mp)}(\tau) \bar{y}_{0}^{(\mp)^{\prime}}\left(t_{0}\right)+\Delta f_{t}^{(\mp)}(\tau)\right) t_{j}+\bar{G}_{j}^{(\mp)}(\tau) .
\end{aligned}
$$

Writing it into block structure given by

$$
\begin{aligned}
\left(\begin{array}{c}
\frac{d Q_{j}^{(\mp)} u}{d \tau} \\
\frac{d Q_{j}^{(\mp)} v}{d \tau}
\end{array}\right)= & \left(\begin{array}{ll}
F_{11}^{(\mp)}(\tau) & F_{12}^{(\mp)}(\tau) \\
F_{21}^{(\mp)}(\tau) & F_{22}^{(\mp)}(\tau)
\end{array}\right)\left(\begin{array}{l}
Q_{j}^{(\mp)} u(\tau) \\
Q_{j}^{(\mp)} v(\tau)
\end{array}\right) \\
& +\left(\begin{array}{c}
\psi_{1}^{(\mp)}(\tau) \\
\psi_{2}^{(\mp)}(\tau)
\end{array}\right),
\end{aligned}
$$

we obtain

$$
\begin{aligned}
& Q_{j}^{(-)} u=H^{(-)}(\tau)\left(\Delta_{j}^{0}\right)^{(-)} \Phi^{(-)}(\tau)\left(\Phi^{(-)}(0)\right)^{-1} \\
& +H^{(-)}(\tau) \int_{0}^{\tau} \Phi^{(-)}(\tau)\left(\Phi^{(-)}(s)\right)^{-1}\left[F_{21}^{(-)}(s)\right. \\
& \cdot \int_{-\infty}^{s} \Psi^{(-)}(\tau)\left(\Psi^{(-)}(\xi)\right)^{-1} \\
& \cdot\left(\psi_{1}^{(-)}(\xi)-H^{(-)}(\xi) \psi_{2}^{(-)}(\xi)\right) d \xi \\
& \left.\quad+\psi_{2}^{(-)}(s)\right] d s+\int_{-\infty}^{\tau} \Psi^{(-)}(\tau)\left(\Psi^{(-)}(s)\right)^{-1} \\
& \cdot\left(\psi_{1}^{(-)}(s)-H^{(-)}(s) \psi_{2}^{(-)}(s)\right) d s, \\
& Q_{j}^{(-)} v=\left(\Delta_{j}^{0}\right)^{(-)} \Phi^{(-)}(\tau)\left(\Phi^{(-)}(0)\right)^{-1}+\int_{0}^{\tau} \Phi^{(-)}(\tau) \\
& \quad \cdot\left(\Phi^{(-)}(s)\right)^{-1}\left[F_{21}^{(-)}(s) \int_{-\infty}^{s} \Psi^{(-)}(\tau)\left(\Psi^{(-)}(\xi)\right)^{-1}\right. \\
& \quad \cdot\left(\psi_{1}^{(-)}(\xi)-H^{(-)}(\xi) \psi_{2}^{(-)}(\xi)\right) d \xi \\
& \left.\quad+\psi_{2}^{(-)}(s)\right] d s,
\end{aligned}
$$

where $\left(\Delta_{j}^{0}\right)^{(-)}=B z_{j}^{*}-B \bar{z}_{0}^{(-)^{\prime}}\left(t_{0}\right) t_{j}+B \rho_{j}^{(-)}(\tau), H^{(-)}(\tau)=$ $\partial \phi_{u} / \partial Q_{j}^{(-)} v$, while $\Phi^{(-)}(\tau)$ and $\Psi^{(-)}(\tau)$ are the solutions of

$$
\begin{aligned}
& \frac{d Q_{0}^{(-)} u}{d \tau}=\left(F_{21}(\tau) H^{(-)}(\tau)+F_{22}(\tau)\right) Q_{0}^{(-)} u, \\
& \Phi^{(-)}(0)=E_{k}, \\
& \frac{d Q_{0}^{(-)} v}{d \tau}=\left(F_{11}(\tau)-H^{(-)}(\tau) F_{21}(\tau)\right) Q_{0}^{(-)} v, \\
& \Psi^{(-)}(0)=E_{M-k},
\end{aligned}
$$

respectively. Consider

$$
\begin{aligned}
& Q_{j}^{(+)} u=\left(\Delta_{j}^{0}\right)^{(+)} \Phi^{(+)}(\tau)\left(\Phi^{(+)}(0)\right)^{-1}+\int_{0}^{\tau} \Phi^{(+)}(\tau) \\
& \cdot\left(\Phi^{(+)}(s)\right)^{-1}\left[F_{12}^{(+)}(s) \int_{+\infty}^{s} \Psi^{(+)}(\tau)\left(\Psi^{(+)}(\xi)\right)^{-1}\right. \\
& \cdot\left(\psi_{2}^{(+)}(\xi)-H^{(+)}(\xi) \psi_{1}^{(+)}(\xi)\right) d \xi \\
& \left.\quad+\psi_{1}^{(+)}(s)\right] d s, \\
& Q_{j}^{(+)} v=H^{(+)}(\tau)\left(\Delta_{j}^{0}\right)^{(+)} \Phi^{(+)}(\tau)\left(\Phi^{(+)}(0)\right)^{-1} \\
& +H^{(+)}(\tau) \int_{0}^{\tau} \Phi^{(+)}(\tau)\left(\Phi^{(+)}(s)\right)^{-1}\left[F_{12}^{(+)}(s)\right.
\end{aligned}
$$

$$
\begin{aligned}
& \cdot \int_{+\infty}^{s} \Psi^{(+)}(\tau)\left(\Psi^{(+)}(\xi)\right)^{-1} \\
& \cdot\left(\psi_{2}^{(+)}(\xi)-H^{(+)}(\xi) \psi_{1}^{(+)}(\xi)\right) d \xi \\
& \left.+\psi_{1}^{(+)}(s)\right] d s+\int_{+\infty}^{\tau} \Psi^{(+)}(\tau)\left(\Psi^{(+)}(s)\right)^{-1} \\
& \cdot\left(\psi_{2}^{(+)}(s)-H^{(+)}(s) \psi_{1}^{(+)}(s)\right) d s,
\end{aligned}
$$

where $\left(\Delta_{j}^{0}\right)^{(+)}=A z_{j}^{*}-A \bar{z}_{0}^{(+)^{\prime}}\left(t_{0}\right) t_{j}+A \rho_{j}^{(+)}(\tau), H^{(+)}(\tau)=$ $\partial \phi_{v} / \partial Q_{j}^{(+)} u$, while $\Phi^{(+)}(\tau)$ and $\Psi^{(+)}(\tau)$ are solutions of

$$
\begin{aligned}
& \frac{d Q_{0}^{(+)} u}{d \tau}=\left(F_{11}(\tau)+F_{12}(\tau) H^{(+)}(\tau)\right) Q_{0}^{(+)} u, \\
& \Phi^{(-)}(0)=E_{k}, \\
& \frac{d Q_{0}^{(+)} v}{d \tau}=\left(F_{22}(\tau)-H^{(+)}(\tau) F_{12}(\tau)\right) Q_{0}^{(+)} v, \\
& \Psi^{(-)}(0)=E_{M-k},
\end{aligned}
$$

respectively. The system to determine $L_{j} x\left(\tau_{0}\right), R_{j} x\left(\tau_{1}\right)$ is similar to the system to determine $Q_{j}^{(\mp)} x(\tau)$, so we can obtain $L_{j} x\left(\tau_{0}\right)$ and $R_{j} x\left(\tau_{1}\right)$ using the same method. 


\section{The Existence of the Heteroclinic Orbit}

We consider the associated system

$$
\frac{d \widetilde{z}}{d \tau}=f\left(\widetilde{z}, \bar{y}_{0}\left(t_{0}\right), t_{0}\right)
$$

for (25), (26), which coincide with the auxiliary system (6) when $\bar{t}=t_{0}$. Obviously, there are two equilibriums $M_{-}\left(\alpha\left(t_{0}\right), \bar{y}^{(-)}\left(t_{0}\right), t_{0}\right)$ and $M_{+}\left(\beta\left(t_{0}\right), \bar{y}^{(+)}\left(t_{0}\right), t_{0}\right)$ for (64). Whether the step-type solution from $\alpha(t)$ to $\beta(t)$ for problem (2), (3) exists or not largely depends on the existence of the heteroclinic orbit $\Gamma_{M_{-} M_{+}}$for system (64) which connects $M_{-}$ to $M_{+}$.

The following assumptions ensure the existence of such heteroclinic orbit.

$\left(H_{6}\right)$ For fixed $t_{0} \in(0,1)$, suppose that there exist $M-1$ linearly independent first integrals for system (64) given by

$$
\Phi_{j}\left(\widetilde{z}_{1}, \widetilde{z}_{2}, \ldots, \widetilde{z}_{M}, t_{0}\right)=C_{j}, \quad(j=1,2, \ldots, M-1),
$$

where $C_{j}(j=1,2, \ldots, M-1)$ are independent arbitrary parameters.

Then, the orbit passing through $M_{-}\left(t_{0}\right)$ is given by

$$
\begin{aligned}
\Phi_{j}\left(\widetilde{z}_{1}^{(-)}, \widetilde{z}_{2}^{(-)}, \ldots, \widetilde{z}_{M}^{(-)}, t_{0}\right)=\Phi_{j}\left(M_{-}\left(t_{0}\right), t_{0}\right), \\
(j=1, \ldots, M-1) .
\end{aligned}
$$

The orbit passing through $M_{+}\left(t_{0}\right)$ is given by

$$
\begin{aligned}
\Phi_{j}\left(\widetilde{z}_{1}^{(+)}, \widetilde{z}_{2}^{(+)}, \ldots, \widetilde{z}_{M}^{(+)}, t_{0}\right)=\Phi_{j}\left(M_{+}\left(t_{0}\right), t_{0}\right), \\
(j=1, \ldots, M-1) .
\end{aligned}
$$

It is noted that

$$
\begin{aligned}
\Phi_{j}\left(M_{-}\left(t_{0}\right), t_{0}\right)=\Phi_{j}\left(M_{+}\left(t_{0}\right), t_{0}\right) & \\
& (j=1,2, \ldots, M-1) .
\end{aligned}
$$

If (68) hold, a heteroclinic orbit connecting $M_{-}\left(t_{0}\right)$ and $M_{+}\left(t_{0}\right)$ can be obtained. Meanwhile, $t_{0}$ will be determined through (68) under the following assumption. $t_{0}=\bar{t}_{0}$.

$\left(H_{7}\right)$ Suppose that (68) are compatible and have a solution

Under condition $\left(\mathrm{H}_{6}\right)$, there exists a heteroclinic orbit which connects $M_{-}\left(t_{0}\right)$ and $M_{+}\left(t_{0}\right)$.

Let $\tilde{u}^{(\mp)}=\left(\widetilde{z}_{1}^{(\mp)}, \ldots, \widetilde{z}_{k}^{(\mp)}\right)^{T}, \widetilde{v}^{(\mp)}=\left(\widetilde{z}_{k+1}^{(\mp)}, \ldots, \widetilde{z}_{M}^{(\mp)}\right)^{T}$. $\tilde{u}^{(-)}(\tau)=\phi_{u}\left(\widetilde{v}^{(-)}(\tau)\right)$ are $M-k$ dimensional unstable manifolds which pass $M_{-}\left(t_{0}\right)$ and $\widetilde{v}^{(+)}(\tau)=\phi_{v}\left(\widetilde{u}^{(+)}(\tau)\right)$ are $k$ dimensional stable manifolds which pass $M_{+}\left(t_{0}\right)$. Then, $\tilde{u}^{(-)}(0)=\phi_{u}\left(\widetilde{v}^{(-)}(0)\right)$. By $(66)$, we know $\widetilde{v}^{(-)}(0)=$ $\left(z_{k+1}^{*}, \ldots, z_{M}^{*}\right)^{T}$. So $\widetilde{u}^{(-)}(0)=\left(z_{1}^{*-}, \ldots, z_{k}^{*-}\right)^{T}$. Similarly, $\widetilde{v}^{(+)}(0)=\phi_{v}\left(\widetilde{u}^{(+)}(0)\right)$, while $\widetilde{u}^{(+)}(0)=\left(z_{1}^{*}, \ldots, z_{k}^{*}\right)^{T}$ by $(67)$. So $\widetilde{v}^{(+)}(0)=\left(z_{k+1}^{*+}, \ldots, z_{M}^{*+}\right)^{T}$.

Take $z_{i}^{*}=z_{i}^{*-}(i=1,2, \ldots, k)$, and then $\tilde{u}^{(+)}(0)=\widetilde{u}^{(-)}(0)$. Because of the existence of the heteroclinic orbit, we have

$$
\widetilde{v}^{(-)}(0)=\widetilde{v}^{(+)}(0)=\phi_{v}\left(\phi_{u}\left(\widetilde{v}^{(-)}(0)\right)\right) .
$$

In (69), the coefficient matrix for $z_{k+1}^{*}, z_{k+2}^{*}, \ldots, z_{M}^{*}$ is as follows:

$$
\begin{aligned}
& E_{M-k}-\frac{\partial \phi_{v}}{\partial \phi_{u}} \frac{\partial \phi_{u}}{\partial \widetilde{v}^{(-)}(0)}=E_{M-k}-\frac{\partial \phi_{v}}{\partial \widetilde{u}^{(+)}(0)} \frac{\partial \widetilde{u}^{(+)}(0)}{\partial \widetilde{v}^{(-)}(0)} \\
& =E_{M-k}-H^{(+)}(0) H^{(-)}(0) .
\end{aligned}
$$

$\left(H_{8}\right)$ Suppose that $\operatorname{det}\left(E_{M-k}-H^{(+)}(0) H^{(-)}(0)\right) \neq 0$.

According to $\left(H_{6}\right)$, we know that the solutions for the left and the right problem satisfy condition (14). So the federated system for (50)

$$
\frac{d}{d \tau} Q_{j} z=f_{z}(\tau) Q_{j} z+\widetilde{G}_{j}(\tau)
$$

has a solution which satisfies $Q_{j} z(-\infty)=0, Q_{j} z(+\infty)=0$, where

$$
\begin{aligned}
& \widetilde{G}_{j}(\tau) \\
& =f_{y}(\tau) Q_{j} y \\
& \quad+\left(\Delta f_{z}(\tau) \beta^{\prime}\left(t_{0}\right)+\Delta f_{y}(\tau)\left(\bar{y}_{0}\left(t_{0}\right)\right)^{\prime}+\Delta f_{t}(\tau)\right) t_{j} \\
& \quad+\bar{G}_{j}(\tau) .
\end{aligned}
$$

By [16], system (71) has an exponential dichotomy in $R^{-}$and $R^{+} .\left(F Q_{j} z\right)(\tau)=(d / d \tau) Q_{j} z-f_{z}(\tau) Q_{j} z$ is Fredholm with index zero [17]. Because $(d / d \tau) Q_{0} z(\tau) \in \operatorname{Ker} F$, there exist $\psi(\tau)$ which satisfy $\psi(\tau) \in \operatorname{Ker} F^{*}$. If the solution for (71) exists, the necessary and sufficient condition is given by

$$
\begin{aligned}
& \int_{-\infty}^{+\infty} \psi^{*}(t) \widetilde{G}_{j}(\tau) d \tau=\int_{-\infty}^{+\infty} \psi^{*}(t)\left\{f_{y}(\tau) Q_{j} y\right. \\
& +\left(\Delta f_{z}(\tau) \beta^{\prime}\left(t_{0}\right)+\Delta f_{y}(\tau)\left(\bar{y}_{0}\left(t_{0}\right)\right)^{\prime}+\Delta f_{t}(\tau)\right) t_{j} \\
& \left.\quad+\bar{G}_{j}(\tau)\right\} d \tau=0 .
\end{aligned}
$$

We rewrite (73) as follows:

$$
\begin{aligned}
& t_{j} \int_{-\infty}^{+\infty} \psi^{*}(t)\left(\Delta f_{z}(\tau) \beta^{\prime}\left(t_{0}\right)+\Delta f_{y}(\tau)\left(\bar{y}_{0}\left(t_{0}\right)\right)^{\prime}\right. \\
& \left.\quad+\Delta f_{t}(\tau)\right) d \tau=-\int_{-\infty}^{+\infty} \psi^{*}(t)\left\{f_{y}(\tau) Q_{j} y\right. \\
& \left.\quad+\bar{G}_{j}(\tau)\right\} d \tau .
\end{aligned}
$$

$\left(H_{9}\right)$ Suppose that $\int_{-\infty}^{+\infty} \psi^{*}(t)\left(\Delta f_{z}(\tau) \beta^{\prime}\left(t_{0}\right) \quad+\right.$ $\left.\Delta f_{y}(\tau)\left(\bar{y}_{0}\left(t_{0}\right)\right)^{\prime}+\Delta f_{t}(\tau)\right) d \tau \neq 0$.

Under this assumption, $t_{j}$ is completely determined. So far, we have already determined all coefficients of the formal asymptotic solution. 


\section{Existence of a Step-Type Solution}

The formal asymptotic solution of $x^{(-)}(t, \mu)$ is given by

$$
\begin{aligned}
& x^{(-)}(t, \mu) \\
& \quad=\sum_{j=0}^{n} \mu^{j}\left(\bar{x}_{j}^{(-)}(t)+L_{j} x\left(\tau_{0}\right)+Q_{j}^{(-)} x(\tau)\right)+O\left(\mu^{n}\right)
\end{aligned}
$$

and the formal asymptotic solution of $x^{(+)}(t, \mu)$ is given by

$$
\begin{aligned}
x^{(+)} & (t, \mu) \\
& =\sum_{j=0}^{n} \mu^{j}\left(\bar{x}_{j}^{(+)}(t)+Q_{j}^{(+)} x(\tau)+R_{j} x\left(\tau_{1}\right)\right)+O\left(\mu^{n}\right) .
\end{aligned}
$$

Substituting (75), (76) into the left problem and the right problem, respectively, completely similar to the previous calculation process, we can obtain the systems to determine all the coefficients of the asymptotic expansions. It is noted that $t^{*}$ is given by

$$
t^{*}=t_{0}+\mu t_{1}+\mu^{2} t_{2}+\cdots+\mu^{n}\left(t_{n}+\delta_{t}\right),
$$

while

$$
\begin{array}{r}
x_{p}^{*}(\mu)=x_{p 0}^{*}+\mu x_{p 1}^{*}+\cdots+\mu^{n}\left(x_{p n}^{*}+\delta_{p}^{*}\right), \\
p=k+1, k+2, \ldots, M .
\end{array}
$$

Obviously, the system to determine $Q_{j}^{(\mp)} x(\tau)(j=0,1, \ldots, n)$ is the same as the front. When $j=n$, we only need to change $x_{n}^{*}$ into $x_{n}^{*}+\delta_{t}$ in (47), (52).

Let

$$
U\left(t^{*}, \mu\right)=x^{(-)}\left(t^{*}, \mu\right)-x^{(+)}\left(t^{*}, \mu\right),
$$

where $U=\left(U_{1}, U_{2}, \ldots, U_{M}\right)$. Since $L_{j} x\left(\tau_{0}\right)$ and $R_{j} x\left(\tau_{1}\right)$ at $t=t^{*}$ are both exponentially small quantities, without loss of generality, we can regard $U\left(t^{*}, \mu\right)$ as follows:

$$
\begin{array}{r}
U\left(t^{*}, \mu\right)=\sum_{j=0}^{n} \mu^{j}\left[\left(\bar{x}_{j}^{(-)}\left(t^{*}\right)+Q_{j}^{(-)} x(0)\right)\right. \\
\left.-\left(\bar{x}_{j}^{(+)}\left(t^{*}\right)+Q_{j}^{(+)} x(0)\right)\right]+O\left(\mu^{n+1}\right) .
\end{array}
$$

In terms of the boundary value conditions of the left and right associated problems, we have $A z^{(+)}\left(t^{*}, \mu\right)=A z^{(-)}\left(t^{*}, \mu\right)$. Then (80) yields

$$
U_{1}\left(t^{*}, \mu\right)=U_{2}\left(t^{*}, \mu\right)=\cdots=U_{k}\left(t^{*}, \mu\right)=0 .
$$

In the same time,

$$
\begin{aligned}
& U_{p}\left(t^{*}, \mu\right)=\sum_{j=0}^{n} \mu^{j}\left[\left(\bar{z}_{p j}^{(-)}\left(t^{*}\right)+Q_{j}^{(-)} z_{j}(0)\right)\right. \\
& \left.\quad-\left(\bar{z}_{p j}^{(+)}\left(t^{*}\right)+Q_{j}^{(+)} z_{p}(0)\right)\right]+O\left(\mu^{n+1}\right)=\left(z_{p n}^{(-)}\left(t^{*}\right)\right. \\
& \left.+Q_{n}^{(-)} z_{p}(0)\right)-\left(z_{p n}^{(+)}\left(t^{*}\right)+Q_{n}^{(+)} z_{p}(0)\right) \\
& \quad+O\left(\mu^{n+1}\right) .
\end{aligned}
$$

When $p$ takes its value from $k+1$ to $M$, (82) can be written as

$$
\begin{aligned}
& \left(E_{M-k}-H^{(+)}(0) \dot{H}^{(-)}(0)\right)\left(\begin{array}{c}
\delta_{k+1}^{*} \\
\vdots \\
\delta_{M}^{*}
\end{array}\right)-H^{(+)}(0) \\
& \cdot \int_{-\infty}^{0}\left(\Psi^{(-)}(s)\right)^{-1}\left(\psi_{1 \delta_{t}}^{(-)}(s)-H^{(-)}(s) \psi_{2 \delta_{t}}^{(-)}(s)\right) d s \\
& -\int_{+\infty}^{0}\left(\Psi^{(+)}(s)\right)^{-1}\left(\psi_{2 \delta_{t}}^{(+)}(s)-H^{(+)}(s) \psi_{1 \delta_{t}}^{(+)}(s)\right) d s \\
& +O\left(\mu^{n+1}\right),
\end{aligned}
$$

where $\psi_{1 \delta_{t}}^{(-)}(\tau)$ and $\psi_{2 \delta_{t}}^{(-)}(\tau)$ are the first $k$ components and the rest of components of

$$
\begin{aligned}
& \left(\Delta f_{z}^{(-)}(\tau) \alpha^{\prime}\left(t_{0}\right)+\Delta f_{y}^{(-)}(\tau)\left(\bar{y}_{0}^{(-)}\left(t_{0}\right)\right)^{\prime}+\Delta f_{t}^{(-)}(\tau)\right) \\
& \quad \cdot \delta_{t}
\end{aligned}
$$

respectively. $\psi_{1 \delta_{t}}^{(+)}(\tau)$ and $\psi_{2 \delta_{t}}^{(+)}(\tau)$ are the first $k$ components and the rest of the components of

$$
\begin{aligned}
& \left(\Delta f_{z}^{(+)}(\tau) \beta^{\prime}\left(t_{0}\right)+\Delta f_{y}^{(+)}(\tau)\left(\bar{y}_{0}^{(+)}\left(t_{0}\right)\right)^{\prime}+\Delta f_{t}^{(+)}(\tau)\right) \\
& \quad \delta_{t},
\end{aligned}
$$

respectively.

By $\left(H_{8}\right)$, there exist $\delta_{k+1}^{*}, \delta_{k+2}^{*}, \delta_{M}^{*}$, such that (83) is equal to zero. Namely, (82) is equal to zero. Then, we obtain a steptype contrast structure at the neighborhood of $t^{*}$. Similarly, we can prove $y^{(-)}\left(t^{*}, \mu\right)=y^{(+)}\left(t^{*}, \mu\right)$. In summary, we have the following result.

Theorem 1. Suppose that $\left(H_{1}\right)-\left(H_{9}\right)$ hold. Problem (2), (3) has a step-type contrast structure solution $x(t, \mu)$. Moreover, the following asymptotic expansion holds:

$$
\begin{aligned}
& x(t, \mu) \\
& = \begin{cases}\sum_{i=0}^{n} \mu^{i}\left(\bar{x}_{i}^{(-)}(t)+L_{i} x\left(\tau_{0}\right)+Q_{i}^{(-)} x(\tau)\right)+O\left(\mu^{n}\right), & 0 \leq t \leq t^{*} ; \\
\sum_{i=0}^{n} \mu^{i}\left(\bar{x}_{i}^{(+)}(t)+Q_{i}^{(+)} x(\tau)+R_{i} x\left(\tau_{1}\right)\right)+O\left(\mu^{n}\right), & t^{*} \leq t \leq 1 .\end{cases}
\end{aligned}
$$

\section{Conflict of Interests}

Authors declare that there is no conflict of interests regarding the publication of this paper.

\section{Acknowledgments}

The authors acknowledge the following: (1) the National Natural Science Funds (no. 11501236); (2) the National Natural Science Funds (no. 11471118); (3) the National Natural Science 
Funds (nos. 30921064, 90820307) supported by Knowledge Innovation Project in the Chinese Academy; (4) Department of Mathematics, Shanghai Key Laboratory of PMMP, East China Normal University, 500 Dongchuan Road, Shanghai 200241, China.

\section{References}

[1] V. F. Butuzov and A. B. Vasileva, "Asymptotic behavior of a solution of contrasting structure type," Mathematical Notes, vol. 42, no. 2, pp. 956-961, 1987.

[2] A. S. Aedeev and A. B. Vasileva, "On a contrast structure of step type for a system of two second-order singularly perturbed equations," Computational Mathematics and Mathematical Physics, vol. 36, no. 5, pp. 75-89, 1996.

[3] A. B. Vasil'eva, "Contrast structures of step-like type for a second-order singularly perturbed quasilinear differential equation," Computational Mathematics and Mathematical Physics, vol. 35, no. 4, pp. 520-531, 1995.

[4] M. A. Davydova, "On contrast structure in a system of singularly perturbed equations," Computational Mathematics and Mathematical Physics, vol. 41, no. 7, pp. 1026-1037, 2001.

[5] A. B. Vasileva, "Inner layer in the boundary problem for a system of two singularly perturbed equations order with the same procedure singularity," Computational Mathematics and Mathematical Physics, vol. 41, no. 7, pp. 1067-1077, 2001.

[6] A. F. Wang and M. K. Ni, "The interior layer for a nonlinear singularly perturbed differential-difference equation," Acta Mathematica Scientia, vol. 32, no. 2, pp. 695-709, 2012.

[7] A. F. Wang and M. K. Ni, "Spike-type contrast structures for a nonlinear singularly perturbed second-order equation," Acta Mathematic Scientia, vol. 29, no. 1, pp. 208-216, 2009.

[8] F. Xie, Z. Y. Jin, and M. K. Ni, "On the spike-type contrast structure of a second-order semi-linear differential equation with integral boundary condition," Electronic Journal of Qualitative Theory of Differential Equations, vol. 62, pp. 1-14, 2010.

[9] A. B. Vasileva, "Contrast structure in the three systems of singularly perturbed," Computational Mathematics and Mathematical Physics, vol. 39, no. 12, pp. 2007-2018, 1999.

[10] X.-B. Lin, "Construction and asymptotic stability of structurally stable internal layer solutions," Transactions of the American Mathematical Society, vol. 353, no. 8, pp. 2983-3043, 2001.

[11] J. K. Hale and X.-B. Lin, "Multiple internal layer solutions generated by spatially oscillatory perturbations," Journal of Differential Equations, vol. 154, no. 2, pp. 364-418, 1999.

[12] W. S. Liu, "Geometric singular perturbations for multiple turning points: invariant manifolds and exchange lemmas," Journal of Dynamics and Differential Equations, vol. 18, no. 3, pp. 667-691, 2006.

[13] M. K. Ni and Z. M. Wang, "On step-like contrast structure of singularly perturbed systems," Boundary Value Problems, vol. 2009, Article ID 634324, 17 pages, 2009.

[14] M. K. Ni and Z. M. Wang, "On higher-dimensional contrast structure of singularly perturbed Dirichlet problem," Science China Mathematics, vol. 55, no. 3, pp. 495-507, 2012.

[15] A. B. Vasileva and V. F. Butuzov, Asymptotic Expansions of Singularly Perturbed Differential Equations, Nauka, Moscow, Russia, 1973.

[16] W. A. Coppel, Dichotomies in Stability Theory, vol. 629 of Lecture Notes in Mathematics, Springer, Berlin, Germany, 1978.
[17] K. J. Palmer, "Exponential dichotomies and transversal homoclinic points," Journal of Differential Equations, vol. 55, no. 2, pp. 225-256, 1984. 


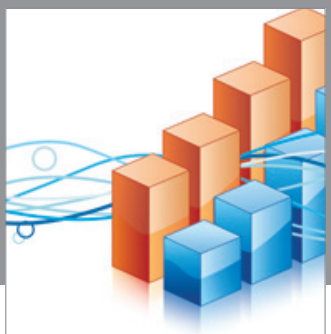

Advances in

Operations Research

vatem alat4

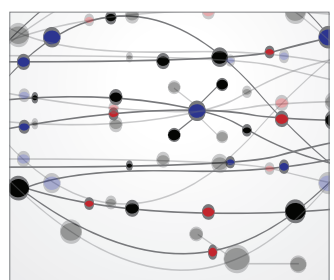

\section{The Scientific} World Journal
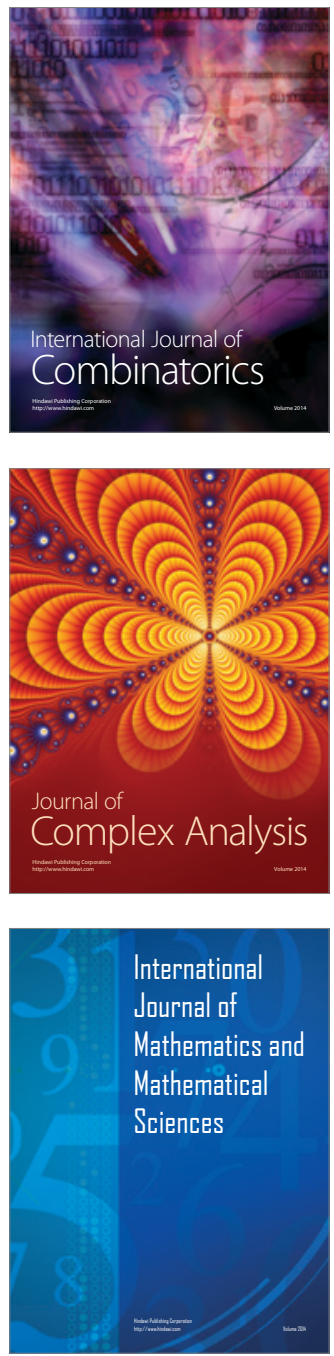
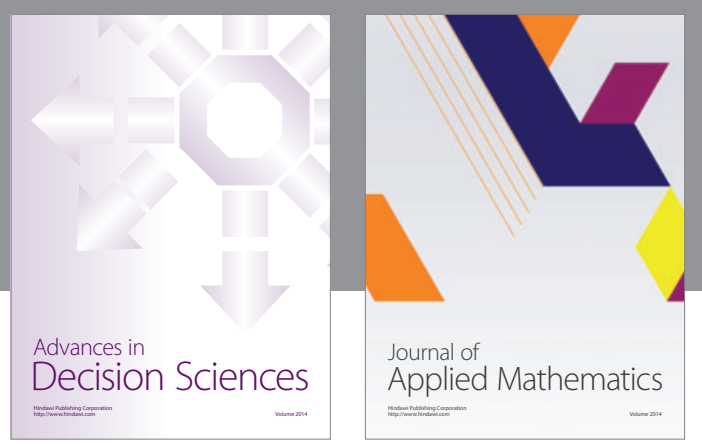

Algebra

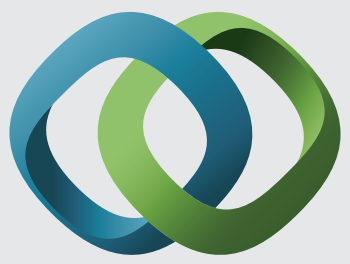

\section{Hindawi}

Submit your manuscripts at

http://www.hindawi.com
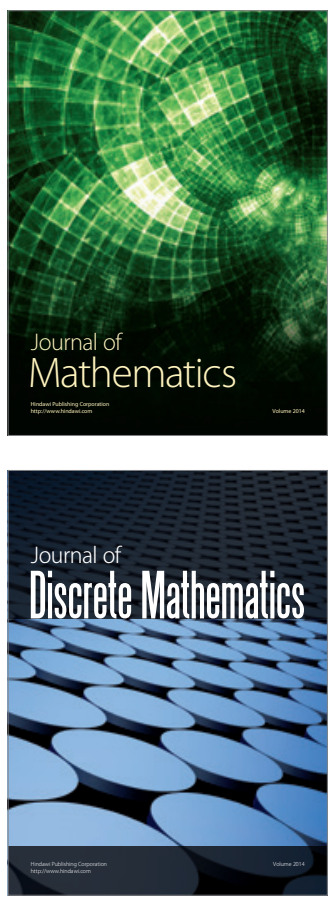

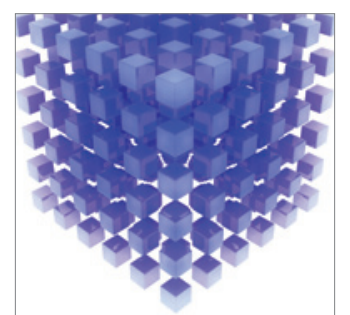

Mathematical Problems in Engineering
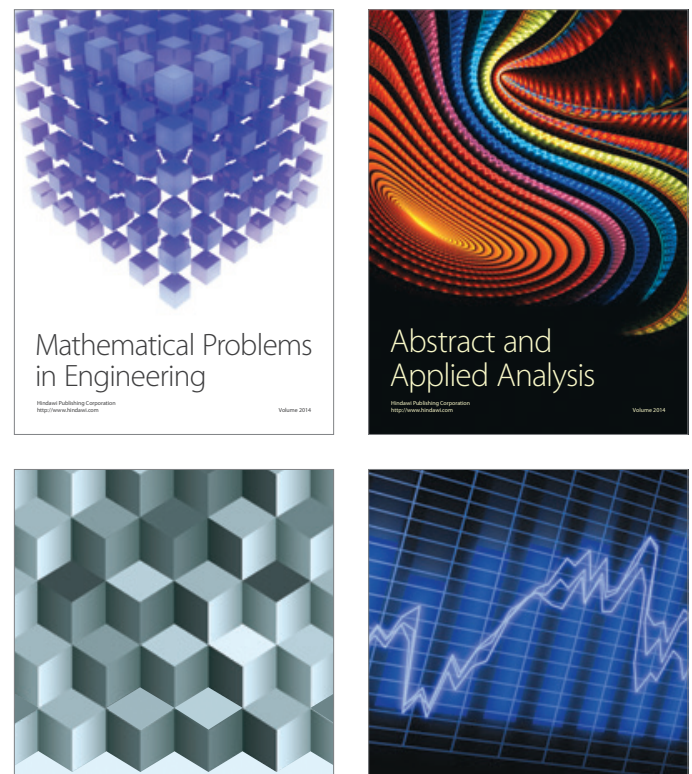

Journal of

Function Spaces

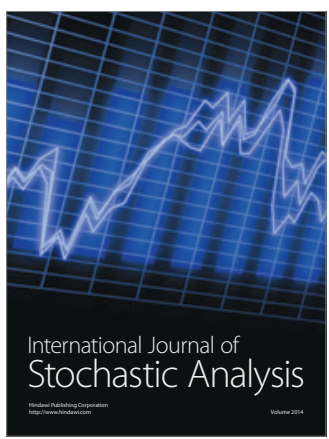

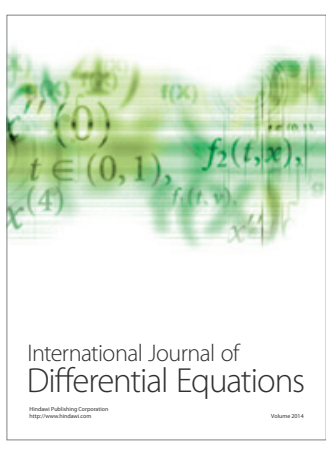
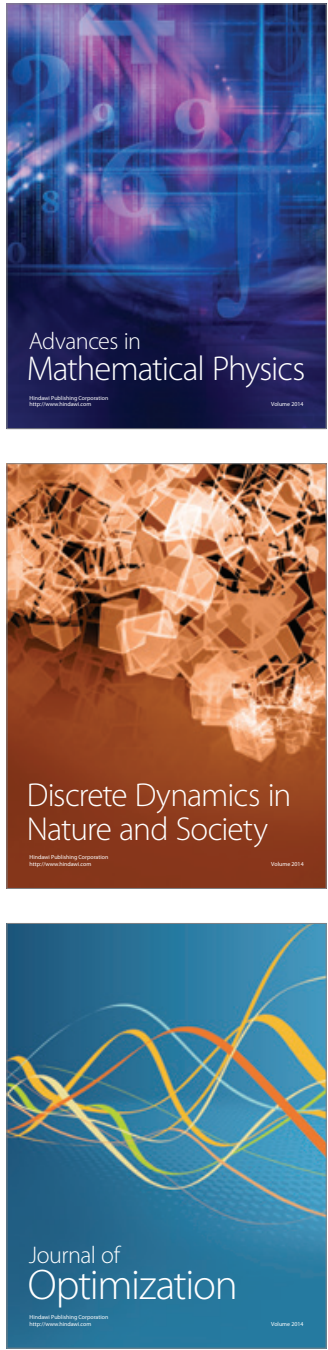